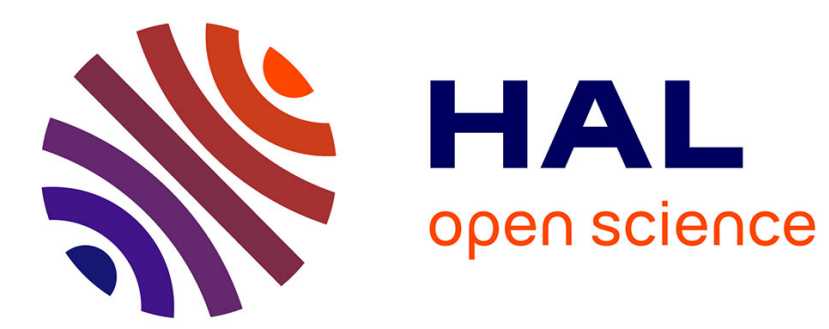

\title{
Chassez le naturel
}

Georges Bertrand

\section{To cite this version:}

Georges Bertrand. Chassez le naturel. Espace Géographique, 1989, La géographie et ses enseignements, 18 (2), pp.102 - 105. 10.3406/spgeo.1989.2854 . hal-02570265

\section{HAL Id: hal-02570265 https://hal.science/hal-02570265}

Submitted on 11 May 2020

HAL is a multi-disciplinary open access archive for the deposit and dissemination of scientific research documents, whether they are published or not. The documents may come from teaching and research institutions in France or abroad, or from public or private research centers.
L'archive ouverte pluridisciplinaire HAL, est destinée au dépôt et à la diffusion de documents scientifiques de niveau recherche, publiés ou non, émanant des établissements d'enseignement et de recherche français ou étrangers, des laboratoires publics ou privés. 


\section{Chassez le naturel}

Georges Bertrand

\section{Citer ce document / Cite this document :}

Bertrand Georges. Chassez le naturel. In: Espace géographique, tome 18, $n^{\circ} 2,1989$. La géographie et ses enseignements. pp. 102-105;

doi : https://doi.org/10.3406/spgeo.1989.2854

https://www.persee.fr/doc/spgeo_0046-2497_1989_num_18_2_2854

Fichier pdf généré le 03/01/2019 
Systèmes analogues, non pas systèmes identiques. Il y a toute la liberté contingente et créatrice de l'homme entre les deux domaines. Mais ce qui dans les systèmes sociaux est projet -- projets et enjeux multiples - a son pendant dans les systèmes physiques: le retour à l'équilibre. Ici, «tout se passe comme si...» les phénomènes observés tendaient vers un steady state, et là, il s'agit soit de systèmes véritablement finalisés et intentionnels de type «téléologique», soit de systèmes inconscients et non intentionnels de type «téléonomique» (4).
L'unité de la géographie, je la placerais à cette série de convergences systémiques. De toute évidence, elle n'aveugle personne. Et pourtant une intuition chevillée au cœur me pousse à choisir cette voie traversière du paradigme dominant. Fourvoiement?-Juillet 1989.

(4) Cf. par exemple, H. Atlan, Création et créativité, 1986, p. 122.

\section{CHASSEZ LE NATUREL...}

Hier on a voulu chasser le naturel de la Géographie. Aujourd'hui...il revient au galop. Sachons chasser le naturel...

Les remarques qui suivent, vite rassemblées pour la circonstance, ne constituent pas une contribution de fond. Mais elles sont une position de thèse. Elles ne datent pas d'aujourd'hui. Depuis plus de vingt ans, elles alimentent la réflexion et les pratiques du CIMA-UA 366. Elles préfigurent un ouvrage en cours de rédaction. Et elles ouvrent un débat à l'occasion du colloque «La Géographie physique, une science de l'environnement?» qui devrait se tenir à Toulouse en 1991.

Ne pas prendre prétexte des difficultés actuelles pour conclure à la disqualification définitive de la Géographie physique. Certes, la crise est ancienne, profonde et, dans sa dérive actuelle, irréversible. Ne pas la traiter comme un épiphénomène universitaire lié à des erreurs (graves) de transmission du savoir et à quelques aberrations individuelles. Ne pas se contenter, comme d'habitude, de collages périphériques et de bricolages hâtifs. Tout n'est pas à rejeter. Mais tout est à repenser: la finalité et le champ, la méthode et la pratique. En commençant par le commencement: l'épistémologie.

\section{Georges BERTRAND}

CIMA (UA 366 CNRS et Université de Toulouse-Le Mirail)

\section{La Nature et la Géographie physique: un double défi au seuil de la modernité}

Le pari est de faire entrer la Géographie physique dans le mouvement scientifique et culturel contemporain comme une science de l'environnement naturel des sociétés. En jouant sur deux points d'ancrage.

1.1. Le retour en puissance de la Nature et à la Nature. Alors que la société industrielle avait occulté la Nature et minimisé ses effets, la société post-industrielle est confrontée à de multiples avatars naturels ou dérivés qui resituent la Nature au cœur du processus économique et culturel. Or les sciences classiques, géologiques ou biologiques, économiques ou sociales, ne sont pas à même de répondre à une complexité qui les dépasse, aux confins encore mal explorés du système social et du système naturel.

1.2. La Géographie physique occupe, de fait, une situation stratégique à la charnière entre la Nature et la Société. Elle correspond à un seuil épistémologique de première grandeur. Tiraillée par ses contradictions internes ou monopolisée par la Géographie humaine et régionale, elle n'a pas su l'exploiter. Aujourd'hui, la Géographie physique sera une discipline d'interface... ou elle ne sera plus rien. 


\section{L'indispensable rupture épistémologique}

Cette Géographie physique en gestation ne doit pas se couper de son patrimoine. Mais elle ne peut avancer dans les seules perspectives ouvertes par Paul Vidal de la Blache, Emmanuel de Martonne ou Pierre Birot, qui représentent des étapes précieuses, mais historiquement datées. L'évolution de plus en plus rapide des lieux, des connaissances et des sensibilités nous place en état d'accélération épistémologique et de «manque» méthodologique. Et s'adapter à s'adapter n'est pas, pour l'instant, le propre du géographe français!

2.1. La Nature n'est plus ce qu'elle était et là où elle était. D'abord, la Nature n'est plus le symbole de l'ordre, de l'équilibre, du renouvelable et de l'inépuisable. La Nature-ressource est «finie», largement «non renouvelable» à l'échelle historique, fragile, déjà pas mal «dénaturée» et parfois «catastrophique». Elle entre, par la force des choses, dans le calcul et la prévision économique. Les sensibilités en sont exacerbées et l'écologisme est devenu une dimension de la vie politique et culturelle, alors que les géographes sont restés professionnellement peu sensibles à ces nouveaux comportements psycho-sociaux.

De plus, la Nature s'est urbanisée, au propre comme au figuré, et elle nous donne une vision inversée de la campagne. La Nature est autant à rechercher dans l'artefact urbain, parc ou «friche industrielle», et dans les broussailles des «terres gelées», que dans le rural agroindustriel. La cuesta qui enracine le village et découpe le finage en terroirs, raconte une histoire, spécifie des contraintes, mais s'efface devant d'autres données qui échappent au site. La Nature ne relève plus de l'«ordre éternel des champs».

2.2. Il faut rompre l'enfermement épistémologique de la Géographic physique française. Sur le plan méthodologique et surtout technique, la Géographie physique a depuis longtemps, et très tôt, emprunté aux sciences de la Nature, et en particulier à la Géologie. Mais la curiosité n'est pas allée beaucoup plus loin, assez peu vers l'Ecologie et encore moins vers les sciences sociales, qui ont pourtant à débattre des questions de nature: Histoire, Préhistoire, Ethnologie, Anthropologie etc. La Géographie physique a encore plus de mal à se situer par rapport au mouvement général des idées et des théories: structuralisme linguistique et ethnologique, théorie des ensembles, analyse de système et écologie de synthèse, réductionnisme biologique, grandes synthèses «éco-socio»... à la Edgar Morin. Comment, avec aussi peu de culture scientifique, prétendre maîtriser la complexité d'un fait ou d'une méthode?

2.3. Il faut prendre en compte la dimension culturelle de la Nature. Fidèle à l'image des sciences de la Nature du $\mathrm{XIX}^{\mathrm{e}}$ siècle, la Géographie physique en est restée, pour l'essentiel, à une approche positiviste et souvent mécaniste de la Nature, jouant très mal de son statut de science sociale. Or, la dimension culturelle de la Nature, à laquelle l'Ecologie n'accédera jamais, est une chance supplémentaire pour la Géographie physique. Le paysage en est l'intercesseur privilégié avec d'autres notions ou concepts «mixtes» tels que le territoire, la ressource, la fertilité, le patrimoine, le vécu et le perçu, toutes les symboliques et tous les tabous qui spécifient et bornent l'environnement naturel des sociétés.

\section{3. (Re)définir un champ disciplinaire: un paradigme socio-naturaliste}

3.1. Une stratégie d'interface. Comment rendre compte du «milieu naturel» en lui-même et pour luimême, dans sa réalité historique, tel qu'il apparaît plus ou moins transformé par l'anthropisation? Comment rendre compte de ce même «milieu naturel» quand il est conçu et analysé comme un «environnement» par les sociétés qui l'utilisent et le transforment? Cette double finalité, à la fois épistémologique et méthodologique, nous paraît irréductible. Elle fonde la spécificité de la démarche. Il reste à l'aménager pour déterminer les priorités et définir une filière méthodologique.

\subsection{La Géographie physique sera naturaliste ou ne} sera pas. L'Ecologie moderne, par son antériorité et son avance méthodologique, fournit un point d'appui et un exemple. La Géographie physique ne peut être que sa débitrice en ce qui concerne l'analyse du monde vivant au travers de l'écosystème. Il s'agit bien d'un «modèle» dans tous les sens du terme. Mais d'un modèle ambigu, quand il outrepasse les systèmes végétaux et animaux pour lesquels il a été conçu. S'agissant du «milieu naturel», il faut faire appel à un modèle naturaliste ou plus exactement néo-naturaliste. Certes pas le naturalisme descriptif et analytique du XIX $\mathrm{x}^{\mathrm{e}}$ siècle, mais celui qui inspire aujourd'hui quelques travaux d'anthropologues et d'ethno-botanistes et qui essaie d'appréhender la complexité fonctionnelle d'un milieu naturel.

3.3. La Nature doit être saisie au cour du social. Comme le proposent concrètement certaines de ces synthèses anthropo-ethnologiques, le modèle naturel se construit à partir de la pratique et de la perception sociales qui lui donnent sa cohérence. Ce postulat social n'est pas contradictoire avec le précédent, qui est d'ordre naturaliste. Le premier finalise le système que le second analyse dans sa structure et dans son fonctionnement. Par exemple, cette forêt est une ancienne réserve seigneuriale traitée en futaie jardinée qui favorise l'accroissement du sapin aux dépens du hêtre et qui, aujourd'hui, sousexploitée, donne des signes de sénescence («tables», chablis, parasitages).

3.4. Le mouvement de la Nature dans le mouvement de l'Histoire. L'étude d'un rapport, ici entre Nature et Société, ne peut que se développer dans le temps, ici 
dans l'Histoire. D'une part, il faut «historiser» l'étude de la Nature pour mieux en appréhender l'évolution récente qui, depuis la révo:ution agricole néolithique, est étroitement dépendante de l'évolution des sociétés rurales et industrielles et de leurs techniques (défrichement, drainage, exploitation des énergies fossiles et des matières premières, pollutions, etc.). D'autre part, sans pour autant restreindre l'Histoire de la Nature à la période préhistorique et historique, il ne paraît pas être très utile de faire remonter l'analyse naturaliste bien au-delà de la mise en place des milieux actuels, au-delà des périodes froides du Quaternaire. On constate la planité de la surface d'érosion post-hercynienne, on n'étudie pas en tant que tel le processus de la pénéplanation!

\section{L'autonomie conceptuelle et méthodologique}

Dépasser le débat géographico-géographique. Les relations de la Géographie physique avec la Géographie humaine et régionale ne sont que de médiocres querelles de ménage, elles ne s'améliorent pas dans un face à face à bout d'arguments. La situation n'a été débloquée que par des échanges interdisciplinaires, par exemple avec la Botanique ou avec l'Histoire. Il n'y a pas de «redéploiement» (R. Brunet) sans élargissement du débat.

- Admettre, une bonne fois pour toutes, que la Géographie physique n'est plus, et n'aurait jamais dû être, un passage obligé pour la Géographie humaine et régionale. L'introduction ou le tableau de géographie physique qui précède beaucoup d'analyses géographiques n'est le plus souvent qu'un ornement superfétatoire, voire une pure ineptie, qui ne pose et surtout ne résout aucun problème concret, et introduit en plus une indélébile contamination déterministe.

- Revendiquer par contre l'autonomie conceptuelle et méthodologique de la Géographie physique, acquise par l'interactivité avec les autres sciences de la Société et de la Nature. Cette autonomie est à définir par rapport à la discipline géographique... peut-être dans l'attente d'une autre solution, qui doit être scientifique avant que d'être institutionnelle. Par contre, la Géographie physique a tout à gagner en se maintenant dans le cadre conceptuel et institutionnel des Sciences sociales, ce qui lui assure une situation privilégiée d'interface.

\section{5. (Re)centrer la méthode}

L'autonomie de la Géographie physique ne sera reconnue que si elle définit une méthode originale permettant de dégager des connaissances pertinentes. C'est loin d'être le cas, du moins en France, où l'on a beaucoup de mal à délimiter le domaine et la fonction du géographe «physicien». La méthode préconisée par le CIMA-UA 366 a déjà été présentée à plusieurs reprises, ainsi que ses applications. Faute de moyens et d'intérêt, elle est restée rudimentaire et souvent allusive. Mais elle occupe une place, au centre de la Géographie physique.

5.1. Le géosystème, un concept central et centralisateur: Le principe est celui de l'analyse de système et le modèle le plus proche en est l'écosystème, où plus exactement les générations d'écosystèmes qui se sont succédé depuis Tansley, Odum, Margalef, Duvigneaud, Lantier, Barbault, etc. Le géosystème n'est pas un élargissement de l'écosystème (qui par le biais de la «landscape ecology» n'a pas donné les résultats escomptés hors du champ propre de la biologie). C'est une autre construction, avec une hiérarchie propre des composants, où interviennent à part entière les éléments non-vivants et les éléments d'origine anthropique.

De plus, le géosystème s'inscrit naturellement dans l'espace (par une grille spécifique: géotope, géofaciès, géocomplexe, etc.) et dans le temps... (donc dans l'Histoire)... ce que l'écosystème n'a jamais réussi à réaliser. Mais le géosystème n'est pas tout et il ne peut se suffire.

5.2. L'interactivité centre-périphérie. La Géographie physique classique, dépourvue de toute référence centrale, n'en finit pas de ne plus exister en se divisant et en se subdivisant. Ce n'est donc qu'à partir d'un centre de gravité que l'on peut envisager le devenir des secteurs spécialisés: Biogéographie, Climatologie et surtout Géomorphologie. L'ensemble repose sur la dialectique entre l'élément et le système, entre la périphérie et le centre. La géographie dite globale (ou intégrée) et les géographies sectorielles ne sont pas contradictoires mais complémentaires. L'un des apports fondamentaux de la Géographie physique «géosystémique» est d'assurer une stratégie de développement périphérique sur l'interface des autres sciences de la Société et de la Nature. C'est le cas exemplaire de la Géomorphologie. Elle est devenue une discipline à part entière, avec la plus large indépendance conceptuelle et méthodologique. Mais avec une finalité qui n'est plus d'ordre géographique. Le géomorphologue étudie en effet les reliefs et les modelés pour eux-mêmes. Il contribue largement, mais sectoriellement, à l'analyse du «milieu naturel» et de l'environnement. Il n'a donc pas à imposer sa problématique, ses concepts et sa finalité de géomorphologue à la Géographie physique. Il lui faut s'adapter à l'analyse géographique comme doit le faire l'écologue ou l'historien. Il faut placer un seuil épistémologique entre la Géographie physique et la Géomorphologie (la Biogéographie, la Climatologie, etc.).

5.3. L'intégration technologique par l'imagerie géographique. La diversité des objets relevant de la Géographie physique a multiplié le recours à des techniques sophistiquées et parfois mal maîtrisées. Souvent, la technique a été confondue avec la méthode, et l'arrivée massive des technologies a encore augmenté la confusion. Pourtant, l'imagerie géographique, cartographique et satellitai- 
re, aujourd'hui en plein développement et en pleine diversification, peut servir de dénominateur commun, à la condition qu'elle soit raisonnée au niveau méthodologique. L'examen du pixel est une excellente introduction à la globalité et à la complexité des phénomènes géographiques, qui sont le plus souvent des «mixtes» Nature-Société comme, par exemple, le paysage.

\section{Promouvoir une formation et une didactique}

Toute tentative de réflexion sur la formation et la didactique conduite sans référence au scientifique et au fondamental, comme c'est actuellement le cas, est vouée à l'échec. On ne peut bien enseigner que les connaissances que l'on maîtrise, c'est-à-dire que l'on peut synthétiser et modéliser, c'est-à-dire simplifier. Or, les contenus de la Géographie physique sont actuellement dispersés et disparates. Il manque un fil conducteur (qui pourrait être le géosystème). D'où l'accumulation et le fatras des manuels. La tectonique des plaques peut ravir les spécialistes et quelques élèves, mais ce n'est qu'un exercice de style hors du champ géographique, et d'ailleurs hors de portée scientifique des géographes qui prétendent l'enseigner.

L'une des missions de la didactique géographique est de participer au développement d'une culture d'interface anthropo-naturaliste qui manque à notre société aux savoirs compartimentés. L'engouement pour la Nature et pour les paysages nous y invite. Mais il faut d'abord que la Géographie physique développe sa propre culture. Dans, hors ou aux marges de la Géographie humaine? Mais que serait la Géographie sans sa «physique»: un vin sans vigne et un bronzage sans soleil!-Mai 1989.

\section{ANNEXE}

\section{PROJET DE COLLOQUE INTERDISCIPLINAIRE «LA GÉOGRAPHIE PHYSIQUE, UNE SCIENCE DE L'ENVIRONNEMENT?»}

Pré-colloque: Printemps 1990 à Paris

Colloque: Printemps 1991 à Toulouse et/ou à Foix

Durée: Trois journées dont une séance de travail sur le terrain

Participants: 40 personnes

Quelques pistes:

1. Histoire-bilan de la Géographie physique

2. La Géographie physique dans la Géographie

3. Les rapports institutionnels et scientifiques avec les Sciences de la Nature et de la Société et dans les grands programmes interdisciplinaires (DGRST, MAB, PIREN etc.)

4. La Géographie physique en quête d'un statut scientifique

5. Quelle didactique pour la Géographie physique?

Chaque thème retenu pendant le pré-colloque sera préparé et animé par un laboratoire ou un groupe de chercheurs ad hoc comprenant obligatoirement des non-géographes.

Pour tout renseignement s'adresser à:

Mme Claude BERTRAND

CIMA-UA 366 CNRS

UFR Institut de Géographie D. Faucher

Université de Toulouse-Le Mirail

5, Allées Antonio Machado

31058 TOULOUSE CEDEX

Tél. 61.41 .11 .05 postes 430 et 361 . 